\title{
Applications of partially quenched chiral perturbation theory
}

\author{
Maarten Golterman ${ }^{\mathrm{a}}$ and Ka Chun Leung ${ }^{\mathrm{b}}$ * \\ a Department of Physics, Washington University, St. Louis, MO 63130, USA. \\ ${ }^{b}$ Institute of Theoretical Physics III, University of Siegen, 57068 Siegen, Germany.
}

Partially quenched theories are theories in which the valence- and sea-quark masses are different. Here, we will discuss the nonanalytic one-loop corrections to some physical quantities, using partially quenched chiral perturbation theory. In particular, we will focus on the results for Goldstone boson masses to illustrate the general features of our calculation.

\section{Partially Quenched Chiral Perturbation Theory (PQChPT)}

We will introduce PQChPT following the formulation in ref. [1]. For simplicity, we will restrict ourselves to the case in which there are 3 quarks $u, d$ and $s$, with masses $m_{u}, m_{d}$ and $m_{s}$ respectively, and a set of $N$ degenerate quarks $q_{S}$ (with common mass $m_{S}$ ) (the arguments can easily be generalized to other cases). We wish to consider lattice computations in which only the $N$ quarks $q_{S}$ contribute as sea quarks. Hence, we add three additional bosonic quarks $\tilde{u}, \tilde{d}$ and $\tilde{s}$ with the same other quantum numbers and masses as their counterparts $u, d$ and $s$, which will cancel the seaquark effects caused by the $u, d$ and $s$ quarks.

If one assumes that, as in unquenched QCD, the strong interactions between these quarks are responsible for chiral symmetry breaking and confinement, one expects Goldstone mesons corresponding to the breakdown of the graded chiral symmetry group $S U_{L}(3+N \mid 3) \times S U_{R}(3+N \mid 3)$ to $S U_{L+R}(3+N \mid 3)$ by the vacuum state (quark masses can be incorporated like in full ChPT). However, the spectrum of Goldstone mesons is enlarged relative to full QCD, and is described by the $(6+N) \times(6+N)$ hermitian matrix

$\Phi=\left(\begin{array}{cc}\phi & \chi_{\tilde{\phi}}^{\dagger} \\ \chi & \tilde{\phi}\end{array}\right)$

where $\phi$ is the $(3+N) \times(3+N)$ matrix of ordinary mesons made from the $3+N$ ordinary

\footnotetext{
* presenter at conference
}

quarks and antiquarks, $\tilde{\phi}$ is the corresponding $3 \times 3$ matrix for bosonic-quark mesons, and $\chi$ is a $3 \times(3+N)$ matrix of fermionic mesons made from a bosonic quark and an ordinary antiquark. One can then construct, using the unitary field $\Sigma$ defined through

$\Sigma \equiv \exp \left(2 i \Phi / f_{p}\right)$

and the diagonal mass matrix $\hat{M}$ with entries

$m_{u}, m_{d}, m_{s}, m_{S}, \cdots, m_{S}, m_{u}, m_{d}, m_{s}$

on the diagonal (where $m_{S}$ occurs $N$ times), the $O\left(p^{2}\right)$ Euclidean partially quenched chiral Lagrangian as:

$$
\begin{aligned}
\mathcal{L}= & \frac{f_{p}^{2}}{8} \operatorname{str}\left(\partial_{\mu} \Sigma \partial_{\mu} \Sigma^{\dagger}\right) \\
& -\frac{f_{p}^{2} \mu_{p}}{4} \operatorname{str}\left(\hat{M} \Sigma+\hat{M} \Sigma^{\dagger}\right) \\
& +\frac{m_{0}^{2}}{6} \Phi_{0}^{2}+\frac{\alpha}{6}\left(\partial_{\mu} \Phi_{0}\right)\left(\partial_{\mu} \Phi_{0}\right),
\end{aligned}
$$

where str denotes the supertrace, and $\Phi_{0} \equiv$ $\operatorname{str}(\Phi)$ is invariant under the chiral symmetry group, and is called super- $\eta^{\prime} . f_{p}$ is the tree-level weak decay constant; $m_{0}$ and $\alpha$ are parameters introduced through the $\Phi_{0}$ terms.

\section{Interplay between $m_{0}$ and $m_{S}$}

The $O\left(p^{2}\right)$ two-point functions can be read off from the Lagrangian eq. (位). In particular, let us consider the $\eta^{\prime}$ two-point function (in the massdegenerate case $m=m_{u}=m_{d}=m_{s}$, and with 
$\alpha=0$ for simplicity). The two-point function reads [1]

$$
\begin{aligned}
\left\langle\eta^{\prime} \eta^{\prime}\right\rangle= & \frac{1}{p^{2}+m_{\pi}^{2}} \\
& +\frac{-m_{0}^{2}}{\left(p^{2}+m_{\pi}^{2}\right)^{2}} \frac{p^{2}+M_{S S}^{2}}{p^{2}+M_{S S}^{2}+N m_{0}^{2} / 3},
\end{aligned}
$$

where the tree-level pion mass-squared $m_{\pi}^{2}=$ $2 \mu_{p} m$, and $M_{S S}^{2}=2 \mu_{p} m_{S}$.

A number of comments is in order. First, the combination $m_{\eta^{\prime}}^{2}=M_{S S}^{2}+N m_{0}^{2} / 3$ is the masssquared of the super- $\eta^{\prime}$ at tree level. Second, in the situation in which the sea quarks are integrated out (hence their dynamical effects are absent), which mathematically corresponds to setting $N=0$ or taking $M_{S S}$ much larger than other relevant energy scales (i.e. external momenta, $m_{0}$ and $m_{\pi}$ ), the last factor in eq. (5) turns into unity and the two-point function degenerates into the form predicted by fully quenched ChPT, with the double-pole term which gives rise to "quenched chiral logarithms." In the partially quenched case, the double pole is also present for $M_{S S} \neq m_{\pi}$. The sea-quark dynamics is encoded in this last factor in which the interplay of the two scales $M_{S S}$ and $m_{0}$ is evident. We will show below in an explicit example how this interplay occurs in physical quantities. Third, one can also envision integrating out the super- $\eta^{\prime}$ when $m_{0}$ is much larger than the other mass scales. This is the limit considered in ref. [2]. Finally, in the unquenched theory, which is obtained by setting $N=3$ and $m_{S}=m$, we obtain for the two-point function

$\left\langle\eta^{\prime} \eta^{\prime}\right\rangle_{f u l l}=\frac{1}{p^{2}+m_{\pi}^{2}+m_{0}^{2}}$,

in which the double pole has disappeared. Again, the $\eta^{\prime}$ decouples from the theory if, in addition, $m_{0}$ is heavy compared to other energy scales. This corresponds to conventional ChPT in which $\eta^{\prime}$ is not explicitly represented.

We thus observe that PQChPT contains different mass scales (all assumed to be below the chiral symmetry breaking scale $\Lambda_{p}$ ): that of the valence quarks and those introduced by partial quenching, i.e. $m_{0}$ and $m_{S}$. In particular, the ratio of $M_{S S}$ and $m_{0}$ is arbitrary. One can develop
PQChPT systematically and it "interpolates" between the fully quenched and unquenched theories. It allows us to assess the situation in which $M_{S S}$ is of the order $m_{0}$, which is likely to be the case in typical partially quenched lattice computations. We have calculated the nonanalytic oneloop corrections for the chiral condensate, weak decay constants, Goldstone-boson masses, $B_{K}$, all with nondegenerate valence-quark masses, and the $K^{+} \rightarrow \pi^{+} \pi^{0}$ decay amplitude with degenerate valence-quark masses. In the following, we will focus only on the one-loop mass predictions in the degenerate valence-quark limit. We refer to ref. [3] for further discussion of other quantities.

\section{Method}

The complete $O\left(p^{4}\right)$ predictions of PQChPT contain contributions from $O\left(p^{4}\right)$ operators whose associated coefficients are largely unknown. To further complicate the matter, there are also one-loop contributions, whose coefficients contain unknown $\eta^{\prime}$ coupling constants, proportional to $\log m_{\eta^{\prime}}^{2}$ coming from $\Phi_{0}$ tadpoles, which thus give rise to a complicated dependence on $m_{S}$ and $m_{0}$. To simplify and still make useful predictions, we adopt a procedure in which only the loop corrections nonanalytic in the valence-quark mass are kept, and each fixed value of the sea-quark mass $m_{S}$ corresponds to a different partially quenched theory. This then allows us to ignore contributions from $\Phi_{0}$ tadpoles. We also expand the results in $m_{\pi}^{2} / m_{\eta^{\prime}}^{2}$ for further simplification. This preserves the property that $\mathrm{PQChPT}$ predictions give the fully quenched results for $M_{S S} \rightarrow \infty$, or the unquenched result for $M_{S S}=m_{\pi}$ (and $m_{0} \rightarrow \infty$ ), when comparing the nonanalytic oneloop corrections. As the fully quenched and unquenched results already existed, this offers a nontrivial check of our results.

The comparison between our results [3] and those of ref. [2], however, is actually more delicate, and deserves some more explanation. In our case, we keep the super- $\eta^{\prime}$, whereas in ref. [2] the super- $\eta^{\prime}$ is integrated out. One would therefore in general expect that in order to "match" the two theories, we would need to adjust the bare parameters. For the quantities considered in ref. 
3], it turns out that, at one loop, all nontrivial adjustments come from $\Phi_{0}$-tadpole contributions. Since we did not include such contributions, we conclude that, for the comparison, no adjustment is needed.

Eventually, one can obtain numerical estimates of one-loop corrections, taking, for example, a value like $700 \mathrm{MeV}$ or $1 \mathrm{GeV}$ (as in refs. [3, 田) for the cutoff $\Lambda_{p}$ in the chiral logarithms. The difference should gives an idea of the size of the unknown contributions from $O\left(p^{4}\right)$ operators and $\Phi_{0}$ tadpoles.

\section{Physical Predictions}

The mass-squared for the pions with degenerate valence quarks at one loop then reads

$$
\left[m_{\pi}^{2}\right]_{1-\mathrm{loop}}=m_{\pi}^{2}\left(1-\frac{2}{3\left(4 \pi f_{p}\right)^{2}} I\right),
$$

where

$$
\begin{aligned}
& I=B+(B+C) \log \frac{m_{\pi}^{2}}{\Lambda_{p}^{2}}, \\
& B=\frac{\left(m_{\pi}^{2}-M_{S S}^{2}\right)\left(m_{0}^{2}-\alpha m_{\pi}^{2}\right)}{m_{\pi}^{2}-M_{S S}^{2}+\frac{N}{3}\left(\alpha m_{\pi}^{2}-m_{0}^{2}\right)},
\end{aligned}
$$

and

$C=-m_{\pi}^{2} \frac{\alpha\left(m_{\pi}^{2}-M_{S S}^{2}\right)^{2}+\frac{N}{3}\left(m_{0}^{2}-\alpha m_{\pi}^{2}\right)^{2}}{\left(m_{\pi}^{2}-M_{S S}^{2}+\frac{N}{3}\left(\alpha m_{\pi}^{2}-m_{0}^{2}\right)\right)^{2}}$,

or, after the expansion in $m_{\pi}^{2} / m_{\eta^{\prime}}^{2}$,

$I=\mathcal{M}^{2}-A m_{\pi}^{2}+\left(\mathcal{M}^{2}-2 A m_{\pi}^{2}\right) \log \frac{m_{\pi}^{2}}{\Lambda_{p}^{2}}$,

and

$$
\begin{aligned}
& \mathcal{M}^{2} \equiv \frac{M_{S S}^{2} m_{0}^{2}}{M_{S S}^{2}+N m_{0}^{2} / 3}=\frac{3 y}{1+N y} M_{S S}^{2} \\
& A \equiv \frac{\alpha M_{S S}^{4}+N m_{0}^{4} / 3}{\left(M_{S S}^{2}+N m_{0}^{2} / 3\right)^{2}}=\frac{\alpha+3 N y^{2}}{(1+N y)^{2}}
\end{aligned}
$$

where $y=\left(m_{0}^{2} / 3\right) / M_{S S}^{2}$.

We will examine the coefficients of the chiral logarithm in eq. (11), which is particularly sensitive to the chiral limit, in more detail. From (partially) quenched lattice data, it is estimated that $m_{0}^{2} / 3$ presumably has a value $m_{K}^{2} / 2 \propto m_{0}^{2} / 3 \stackrel{<}{\sim} m_{K}^{2}\left(m_{K}=496 \mathrm{MeV}\right.$ is the physical kaon mass) [5.6]. Typical lattice compu-

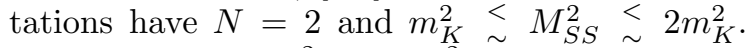
These values of $m_{0}^{2}$ and $M_{S S}^{2}$ correspond to $y$ ranging from $y \approx 1 / 4$ to $y \approx 1$. This leads to $\mathcal{M}^{2} / M_{S S}^{2}=1 / 2$ for $y=1 / 4$ and to $\mathcal{M}^{2} / M_{S S}^{2}=1$ for $y=1$. For $y \rightarrow \infty$ one obtains $\mathcal{M}^{2} / M_{S S}^{2}=$ $3 / 2$. This shows that for relatively heavy sea quarks, there is a clear dependence of the coefficient of the chiral logarithms on $m_{0}^{2}$. (Experience with quenched lattice data [5] indicates that it is hard to fit the chiral logarithms reliably, partially because of the "competition" of $O\left(p^{4}\right)$ coefficients. This may make it difficult to see the $y$ dependence of the chiral logarithms in practice.)

The quantity $A$ also has an effect on the coefficients of the chiral logarithms, in particular for values of the valence-quark mass of order of the sea-quark mass. Taking again $N=2$, we find $A=(8 \alpha+3) / 18$ for $y=1 / 4$ and $A=(\alpha+6) / 9$ for $y=1$, while $A=3 / 2$ for $y \rightarrow \infty$. It is clear that $A$ is more sensitive to the value of $\alpha$ for smaller values of $y$. We note that for $m / m_{S}=1$, our expansion parameter $m_{\pi}^{2} / m_{\eta^{\prime}}^{2}=1 /(1+N y$ ) (for $\alpha=0)$, so that our results may not be reliable for smaller values of $y$. In that case, expression (8) should be used.

We would like to thank Claude Bernard and Steve Sharpe for discussions. This work was supported in part by the DOE.

\section{REFERENCES}

1. C. W. Bernard and M. F. L. Golterman, Phys. Rev. D 49, 486 (1994).

2. S. R. Sharpe, Phys. Rev. D 56, 7052 (1997).

3. M. F. L. Golterman and K.-C. Leung, Phys. Rev. D 57, 5703 (1998).

4. M. F. L. Golterman and K.-C. Leung, Phys. Rev. D 56, 2950 (1997).

5. S. R. Sharpe, Nucl. Phys. B (Proc. Suppl.) 53, 181 (1997).

6. L. Venkataraman and G. Kilcup, heplat/9711006, Phys. Rev. D, in print; R. Burkhalter, for CP-PACS, these proceedings; T. Yoshie, for CP-PACS, these proceedings. 